\title{
Always Contact a Vascular Interventional Specialist Before Amputating a Patient with Critical Limb Ischemia
}

\author{
Rosemarie Met · Mark J. W. Koelemay • \\ Shandra Bipat · Dink A. Legemate · \\ Krijn P. van Lienden · Jim A. Reekers
}

Received: 2 June 2009/ Accepted: 22 July 2009/Published online: 18 August 2009

(c) The Author(s) 2009. This article is published with open access at Springerlink.com

\begin{abstract}
Patients with severe critical limb ischemia (CLI) due to long tibial artery occlusions are often poor candidates for surgical revascularization and frequently end up with a lower limb amputation. Subintimal angioplasty (SA) offers a minimally invasive alternative for limb salvage in this severely compromised patient population. The objective of this study was to evaluate the results of SA in patients with CLI caused by long tibial occlusions who have no surgical options for revascularization and are facing amputation. We retrospectively reviewed all consecutive patients with CLI due to long tibial occlusions who were scheduled for amputation because they had no surgical options for revascularization and who were treated by SA. A total of 26 procedures in 25 patients (14 males; mean age, $70 \pm 15$ [SD] years) were evaluated. Technical success rate was $88 \%$ (23/26). There were four complications, which were treated conservatively. Finally, in 10 of 26 limbs, no amputation was needed. A major amputation was needed in 10 limbs ( 7 below-knee amputations and 3 above-knee amputations). Half of the major amputations took place within 3 months after the procedure. Cumulative freedom of major amputation after 12 months was $59 \%(\mathrm{SE}=11 \%)$. In six limbs, amputation was limited to a minor amputation. Seven patients (28\%) died during follow-up. In conclusion, SA of the tibial arteries seem to be a valuable treatment option to prevent major amputation
\end{abstract}

R. Met $(\bowtie) \cdot$ S. Bipat · K. P. van Lienden · J. A. Reekers Department of Radiology, Academic Medical Center, Meibergdreef 9, 1105 AZ Amsterdam, The Netherlands e-mail: r.met@amc.uva.nl

M. J. W. Koelemay · D. A. Legemate Department of Surgery, Academic Medical Center, Meibergdreef 9, 1105 AZ Amsterdam, The Netherlands in patients with CLI who are facing amputation due to lack of surgical options.

Keywords Subintimal angioplasty - Tibial arteries · Critical limb ischemia

\section{Introduction}

Critical limb ischemia (CLI) is a manifestation of severe peripheral arterial disease, characterized by pain at rest, nonhealing ulcers, or both. Revascularization is necessary to save the limb and this can be performed surgically or percutaneously [1]. When both treatments are possible, endovascular therapy is preferred because of similar clinical outcomes and lower costs [2].

Percutaneous angioplasty of the infrapopliteal arteries has long been regarded as a difficult procedure because of the small caliber of the tibial arteries and the tendency to develop spasm [3, 4]. Over the years experience with percutaneous treatment of infrapopliteal disease has grown, with promising technical and clinical results [5-7]. While short occlusions can be treated percutaneously, most long occlusions still require a surgical approach. Subintimal angioplasty (SA) is a percutaneous alternative to surgery to overcome long occlusions, when a transluminal approach would be technically impossible. Although SA of tibial arteries can result in high limb salvage rates, most studies do not differentiate between severe and mild ischemia and do not state whether a surgical option was still available. As a result, such mixed patient groups show higher limb salvage rates compared with patients with CLI without a surgical option for revascularization.

Patients with end-stage CLI with long and multiple tibial artery occlusions, without options for surgical revascu- 
larization, often end up with an amputation. In such patients, infrapopliteal SA could be an interesting treatment and a last chance to save the patient's limb. This 'percutaneous temporary pedal bypass' was described previously [8].

The purpose of this study was to evaluate whether tibial artery SA in end-stage CLI patients without surgical options an amputation. As a secondary objective of the study, we evaluated the influence of outflow on clinical success.

\section{Methods}

Patients

A retrospective review was performed of all consecutive patients without surgical options for revascularization who underwent a percutaneous vascular intervention of the tibial arteries between 1 August 2006 and 31 December 2008. All patients were considered candidates for amputation due to uncontrollable ischemic rest pain or nonhealing ulcers, necrosis, or gangrene. The indication for amputation was made by the vascular surgeon, in close consultation with the interventional radiologist. To confirm correct patient selection, we reviewed all patients with CLI who presented at our hospital during the study period to verify the indication once more with an expert panel consisting of a vascular surgeon (M.K.) and an interventional radiologist (J.R.). We included only patients with an absolute indication for amputation, i.e., absence of a suitable artery for distal anastomosis. All patients with a clinical presentation showing an option for healing were accepted for an attempt at endovascular revascularization. Only those patients who were having severe gangrene with deep infection, patients who were septic, and patients with severe ischemia who were not ambulatory due to other medical causes underwent primary amputation. All patients were treated for long and multiple tibial artery occlusions. Accompanying poor inflow due to stenoses in the superficial femoral artery (SFA) was not an exclusion criterion. These lesions were treated in the same procedure. Risk factors [9], previous interventions, preintervention vascular assessment, indication, and length of the treated lesion were recorded.

During the study period, 14 patients needed a primary amputation due to severe gangrene with deep infection without any chance of healing. A total of 25 patients were included in the study. One patient was successively treated in both legs, with an interval of 6 months between the two interventions. Therefore, 26 interventions were analyzed. Patient characteristics are reported in Table 1. Fourteen were men and the mean age was $70 \pm 15$ (SD) years.
Table 1 Patient characteristics at assessment $(n=25)$

\begin{tabular}{lc}
\hline Characteristic & Mean $( \pm \mathrm{SD})$ or $n(\%)$ \\
\hline Gender, male & $14(56 \%)$ \\
Age, year & $70.3( \pm 15.2)$ \\
Risk factors & \\
Diabetes mellitus & $16(64 \%)$ \\
Hypertension & $18(72 \%)$ \\
Current or former smoker & $14(56 \%)$ \\
Smoking unknown & $9(36 \%)$ \\
Renal failure & $13(52 \%)$ \\
Hypercholesterolemia & $7(28 \%)$ \\
History of coronary artery disease & $4(16 \%)$ \\
History of stroke & $3(12 \%)$ \\
\hline
\end{tabular}

Note: Hypertension was defined as a diastolic blood pressure $>90 \mathrm{mmHg}$ or being on antihypertensive medication; renal failure was defined as an elevated creatinine level, $>110 \mu \mathrm{mol} / \mathrm{L}$; and hypercholesterolemia was defined as an elevated triglyceride level, $>2.00 \mathrm{mmol} / \mathrm{L}$, or an elevated LDL cholesterol level, $>4.82 \mathrm{mmol} / \mathrm{L}$ [9]

Sixty-four percent had diabetes mellitus and renal failure was present in half of the patients, including four hemodialysis patients (16\%). All patients suffered from atherosclerotic vascular disease. Most limbs were treated because of tissue loss (Table 2). The large number of patients with previous interventions, including contralateral amputation, highlights the severity of arterial obstructive disease in our patient group. In total 10 stenoses and 31 occlusions were treated, of which 3 occlusions and 2 stenoses were located in the femoral artery. All other lesions were located in the lower limb. Most of the occlusions were at least $20 \mathrm{~cm}$ long (Table 3).

Table 2 Limb characteristics at assessment $(n=26)$

\begin{tabular}{ll}
\hline Characteristic & Median (range) or $n(\%)$ \\
\hline Fontaine stage & \\
$\quad$ III, rest pain & $3(12 \%)$ \\
$\quad$ IV, nonhealing ulcer & $23(88 \%)$ \\
Ankle pressure (highest; mmHg) & $70(36-135)$ \\
Toe pressure (mmHg) & $29(0-67)$ \\
Previous vascular intervention, ipsilateral & $15(58 \%)$ \\
Below-knee amputation, contralateral & $7(27 \%)$ \\
\hline
\end{tabular}

Table 3 Length of lesions

\begin{tabular}{llc}
\hline & Stenoses & Occlusions \\
\hline $0-5 \mathrm{~cm}$ & 3 & 5 \\
$5-10 \mathrm{~cm}$ & 4 & 4 \\
$10-20 \mathrm{~cm}$ & 0 & 3 \\
$20-30 \mathrm{~cm}$ & 3 & 14 \\
Total reconstruction (tibial-popliteal-femoral) & 0 & 5 \\
\hline
\end{tabular}




\section{Procedure}

All occlusions were treated by the standard SA technique under local anesthesia [10]. Antiplatelet drugs and oral vitamin $\mathrm{K}$ antagonist were not discontinued prior to the intervention. An antegrade puncture of the ipsilateral common or SFA was performed. The subintimal space was entered proximal to the occlusion and the occluded segment was traversed with a 0.035 -in. guidewire (Terumo, Tokyo). After re-entry into the lumen distal of the occlusion, the subintimal space was dilated with a $3-\mathrm{mm}, 5-\mathrm{Fr}$ PTA balloon. The balloon lengths varied from 8 to $22 \mathrm{~cm}$. Both standard PTA balloons (Opta 5; Cordis Europe, Johnson \& Johnson, Roden, The Netherlands) and highpushibility PTA balloons (Reekross catheter; ClearStream Technologies Ltd., Wexford, Ireland) were used [11]. No stents were used in the tibial arteries. During the intervention, $5000 \mathrm{IU}$ of heparin was administered. After the intervention, every patient was prescribed anticoagulant medication, either an antiplatelet drug or an oral vitamin $\mathrm{K}$ antagonist, if this was already being used for other indications. If the interventionalist judged the flow after the procedure to be slow, then also clopidogrel, $75 \mathrm{mg}$ daily for 3 months, was prescribed. Patients were also treated with intravenous heparin until they were well set on oral anticoagulant therapy according to a standardized protocol [12]. Briefly, the heparin dose was $1000 \mathrm{IU} / \mathrm{h}$ and the duration of administration was dependent on the APTT value, which was determined $6 \mathrm{~h}$ after the procedure. The target APTT level was 2 times the APTT normal value. Figure 1 shows the angiograms of a patient before and after subintimal angioplasty of the popliteal and anterior tibial artery.

\section{Follow-Up}

Clinical follow-up was performed by the vascular surgeon. Follow-up regimens were left to the surgeon's discretion and when clinically indicated. Final study follow-up was done by contacting the patient's general practitioner.

\section{End Points}

The primary end point was limb salvage. Limb salvage was defined as freedom from major amputation, i.e., any amputation above the level of the ankle. Secondary end points included technical success of the procedure, defined as $<30 \%$ final residual stenosis measured at the narrowest point of the vascular lumen, and clinical success, defined as recovery or stabilization of rest pain or ulcers and freedom from minor amputation, defined as any transmetatarsal amputation or amputation of one or more toes. Complications were classified as minor and major [13].

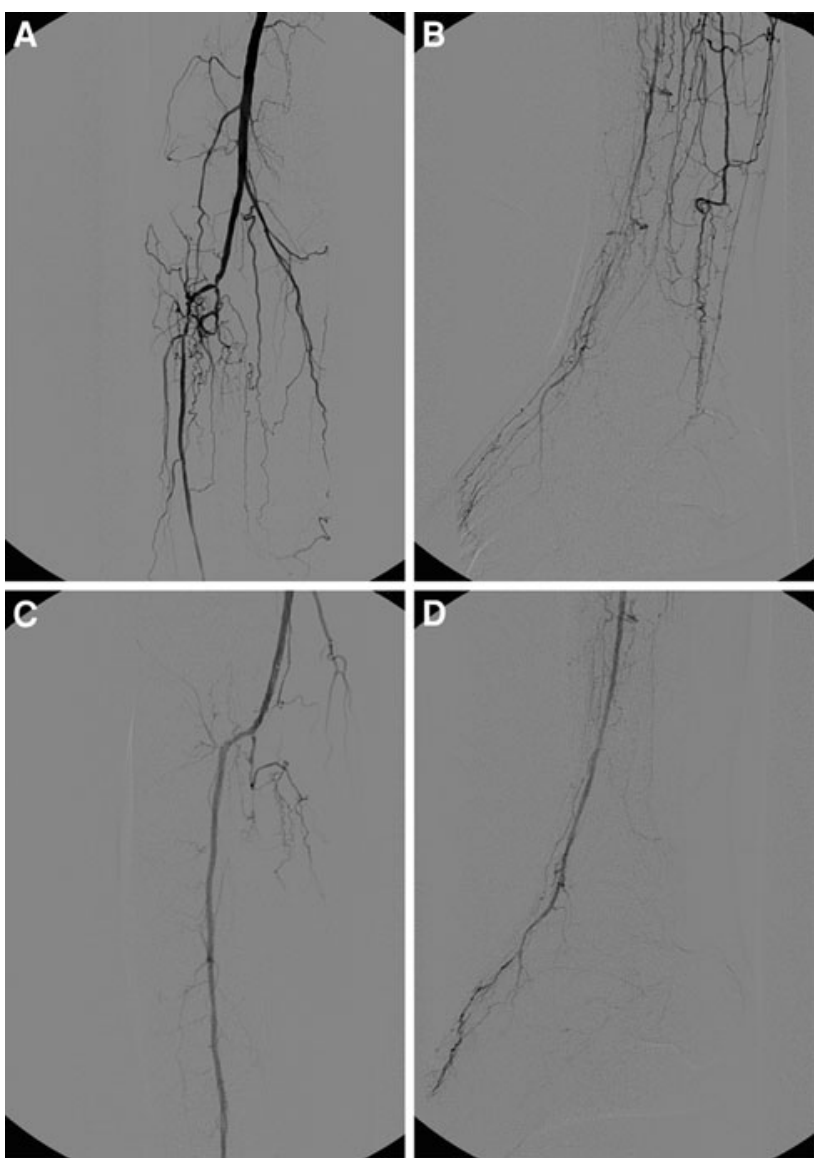

Fig. 1 Angiogram of the lower leg and foot of a patient before $(\mathbf{A}, \mathbf{B})$ and after $(\mathbf{C}, \mathbf{D})$ subintimal angioplasty of the popliteal and anterior tibial artery

To explore any differences in outflow between patients with and those without a major amputation, the before and after intervention angiograms were retrospectively examined by two experienced interventional radiologists (with 6 and 23 years of experience, respectively) who were unaware of the clinical outcome of the patient. The popliteal to distal vessels were assessed according to three categories: 0 , occlusion; 1 , severe vessel wall irregularities and diffuse narrowing; and 2, no or minor vessel wall irregularities and a fully patent lumen. Moreover, we recorded outflow to the foot after intervention, whether there was direct flow to the dorsalis pedis artery, plantar arterial arch, and lateral plantar artery. In the case of disagreement between the two radiologists, the images in question were jointly reviewed and a consensus sought.

\section{Statistical Analysis}

Patient characteristics were summarized using descriptive statistics. We analyzed the entire group as well as the subgroups based on outcome after intervention (patients 
with a major amputation versus other patients). Patientrelated outcomes, like survival and patient characteristics, are presented on a per-patient basis. Procedure-related outcomes, like limb salvage and technical success, are presented on a per-limb basis. For exploring possible differences in inflow (whether or not the SFA was treated) and outflow between patients who improved and those who were amputated, we used the chi-square or Fisher's exact test. Survival and limb salvage were calculated using the Kaplan-Meier method. Agreement between the two radiologists in assessing the angiograms is expressed as weighted kappa values. Statistical analyses were performed using SPSS 15.0 software (SPSS Inc., Chicago, IL, USA).

\section{Results}

\section{Procedure}

All patients were treated by SA for long tibial artery occlusions. In 11 limbs, a subintimal or transluminal revascularization of the SFA was also performed. The intervention was technically successful in 23 of 26 limbs $(88 \%)$. Reasons for failure were calcifications that were too extensive in one patient and lack of peripheral runoff found for re-entry in two patients. Before intervention, 11 patients had all three tibial arteries occluded, 14 patients had one patent tibial artery, and 1 patient had two patent tibial arteries. After intervention, in 1 patient all three tibial

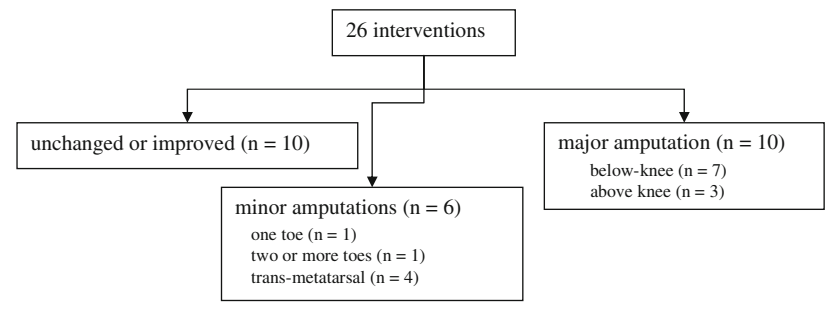

Fig. 2 Clinical outcome arteries were still occluded, 13 patients had one patent tibial artery, and 11 patients had 2 patent tibial arteries. In 1 patient, all three tibial arteries were patent.

\section{Complications}

Complications occurred after 4 procedures. There were 4 minor complications; a dissection, a subsequent local extravasation, and a false aneurysm-all treated conservatively; and a nose bleed, which required insertion of a tampon. There was one major complication: an acute rightsided cardiac failure which required a 2-day admission to the cardiac care unit. Patients were discharged a median of 9 days (range, 0-115 days) after intervention.

The median follow-up was 7 months (range, 43 days27 months). No patient was lost to follow-up. Clinical outcome is shown in Fig. 2. Ten limbs were free of amputation, 6 limbs required a minor amputation, and 10 a major amputation. Of the three patients with an unsuccessful procedure, two ended up with a below-knee amputation and one with a transmetatarsal amputation. Seven patients (28\%) died within 43 days to 13.5 months. The cause of death was unknown in four patients since no autopsy was performed, one died of cardiac failure, one died after a stroke, and one of a sepsis, caused by leg ulcers. The cumulative limb salvage rate after 12 months was $59 \%(\mathrm{SE}=11 \%)$, and the cumulative survival after 12 months $69 \%$ ( $\mathrm{SE}=11 \%$ ). Figure 3 shows the KaplanMeier curves of survival and major amputation. After 1 year 13 patients $(52 \%)$ were alive with a preserved limb.

The agreement of the two interventional radiologists regarding assessment of the arteries before and after treatment was substantial (weighted $\kappa=0.76$; 95\% confidence interval, 0.71-0.82). Before the procedure, the vessels of patients who underwent a major amputation were worse than those of patients who improved or who needed only a minor amputation, with more frequent severe stenosis or occlusion (nonsignificant). After the procedure, especially the anterior tibial artery and the dorsalis pedis
Fig. 3 Kaplan-Meier limb salvage (A; 26 limbs) and survival (B; 25 patients) curves. $S E$ standard error
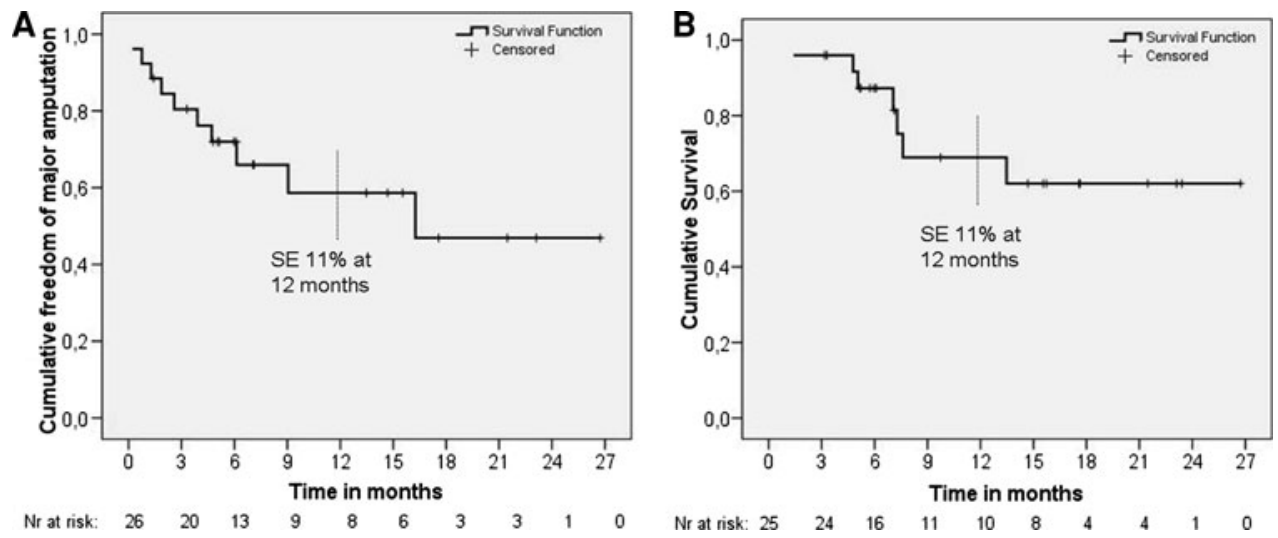
Table 4 Outflow to the arteries of the foot after intervention: comparison between patients who improved after treatment $(n=16)$ and patients who received a major amputation $(n=10)$

\begin{tabular}{llll}
\hline & Direct flow to dorsalis pedis artery & Direct flow to plantar artery & Direct flow to pedal-plantar arch \\
\hline Improved $(n=16)$ & $11(69 \%)$ & $2(13 \%)$ & $5(31 \%)$ \\
Amputation $(n=10)$ & $3(30 \%)$ & $2(20 \%)$ & $0(0 \%)$ \\
$p$ value & 0.11 & 0.63 & 0.12 \\
\hline
\end{tabular}

Note: Levels of significance calculated by means of Fisher's exact test

artery were significantly of better quality in the improved patients $(p=0.059$ and $p=0.005$, respectively).

Concomitant treatment of SFA lesions was not associated with improved outcome. On the contrary, some $60 \%$ of patients with a major amputation had a concomitant SFA revascularization, versus $31 \%$ of patients who did not have a major amputation, although this difference was not statistically significant $(p=0.23)$.

Improved patients had direct flow to the foot via the dorsalis pedis artery after the intervention more often compared to patients who were amputated (Table 4; $p=0.11$, nonsignificant).

\section{Discussion}

In this study, we showed that SA of long tibial occlusions in patients with severe chronic CLI without surgical options for revascularization is technically feasible and has a good clinical success rate. In one-third of patients the clinical status improved or remained unchanged. A major amputation was still needed in nearly one-third of the patients, and a minor amputation in one-fourth. The technical success of the procedure was high, and there were only a few complications, which could be treated conservatively. Based on these results, we think that a patient scheduled for amputation due to end-stage chronic CLI must always be referred to a vascular interventional specialist with experience in lower limb SA.

In the present study, outflow after intervention was associated with clinical outcome. Patients whose limb was saved after intervention more often had direct outflow to the dorsalis pedis artery. There was a trend toward a better clinical outcome in the case of direct flow to the pedalplantar arch after intervention. These results confirm the importance of adequate outflow in both endovascular [10] and surgical revascularization $[1,14-16]$ procedures. This implies that opening of the tibial arteries is not enough; the intervention should also focus on improvement of outflow. New low-profile balloons and dedicated 0.14-in. guidewires, not used in the current study, might help to target this outflow problem. In the present study, we did not perform additional PTA of the foot arteries. However, due to very low flow before the intervention, filling of the foot arteries was nominal. After treatment, the filling increased a lot due to better inflow caused by the SA of the tibial arteries. As a result of this study, we have started using foot artery PTA or SA to establish adequate outflow.

In our study we also found that patients who had undergone concomitant revascularization of the SFA in the same session had a worse clinical outcome, possibly indicating that patients with more extensive disease benefit less from revascularization. The cohort was too small to allow for a multivariate analysis.

In the few studies reporting on SA of the infrapopliteal arteries in patients with CLI [5-7, 17, 18], the technical success rate was high, ranging from 78 to $86 \%$, and comparable to our technical success rate $(88 \%)$. Our complication rate of $15 \%(4 / 26)$ was also in line with these studies. One-year limb salvage in our study was 59\%, versus a reported 1-year limb salvage rate ranging from 81 to $94 \%$. This difference probably reflects patient selection. We included only patients with CLI without options for surgical revascularization requiring amputation. It is known from a previous study, which included patients who were both suitable and unsuitable for a surgical bypass, that unsuitability for surgery was associated with restenosis, reintervention, or amputation [19].

SA of the tibial arteries must be considered among other alternative therapies for end-stage CLI patients, such as spinal cord stimulation [20], treatment with Iloprost [2123], and therapeutic angiogenesis with progenitor cells [24-26].

Some shortcomings of this study should be mentioned. The retrospective nature of our study results in different sources of bias. First, selection bias potentially influenced our results; we only studied a selected sample of a larger group of patients with CLI. Second, retrospective data collection often leads to incomplete data. For example, in our study, pressure measurements were missing for 10 patients. Therefore, our results cannot be supported by objective pressure measurements. Also, follow-up was not performed according to a standard protocol, although we tried to perform follow-up as completely as possible by contacting treating physicians or local general practitioners. The lack of a control series is another limitation of 
this study and reduces the evidence for definite conclusions.

Currently, there are no guidelines for anticoagulant therapy after interventions of the tibial arteries. There is wide consensus that all patients with symptomatic peripheral arterial disease should be treated with anticoagulant therapy [1]. In our study, anticoagulant therapy after the procedure was not prescribed according to a standard protocol. All patients were treated at least with aspirin or a vitamin $\mathrm{K}$ antagonist. After the procedure, the interventionalist judged the speed of flow, and when it was assessed to be slow, clopidogrel, $75 \mathrm{mg}$ daily for 3 months, was prescribed. Again, due to the small size of the cohort, we were not able to analyze the influence of anticoagulant regimen on outcome.

There were no stents placed in the tibial arteries because there is no evidence of benefit of stent placement in comparison with revascularization only.

In conclusion, subintimal angioplasty of the tibial arteries seems to be a valuable treatment option to prevent major amputation in patients with severe CLI without any surgical bypass options, facing amputation. Taking into account the short life expectation of this population, subintimal angioplasty must be considered as the last chance to save the patient's limb. Crucial for the outcome of this kind of last-resort procedure is establishing good outflow for the recanalized vessels.

Open Access This article is distributed under the terms of the Creative Commons Attribution Noncommercial License which permits any noncommercial use, distribution, and reproduction in any medium, provided the original author(s) and source are credited.

\section{References}

1. Norgren L, Hiatt WR, Dormandy JA et al (2007) Inter-society consensus for the management of peripheral arterial disease (TASC II). Eur J Vasc Endovasc Surg 33(Suppl 1):S1-S75

2. Adam DJ, Beard JD, Cleveland T et al (2005) Bypass versus angioplasty in severe ischaemia of the leg (BASIL): multicentre, randomised controlled trial. Lancet 366(9501):1925-1934

3. Bertelè V, Roncaglioni MC, Pangrazzi J et al (1999) Clinical outcome and its predictors in 1560 patients with critical leg ischaemia. Chronic Critical Leg Ischaemia Group. Eur J Vasc Endovasc Surg 18(5):401-410

4. Marston WA, Davies SW, Armstrong B et al (2006) Natural history of limbs with arterial insufficiency and chronic ulceration treated without revascularization. J Vasc Surg 44(1):108-114

5. Ingle H, Nasim A, Bolia A et al (2002) Subintimal angioplasty of isolated infragenicular vessels in lower limb ischemia: long-term results. J Endovasc Ther 9(4):411-416

6. Vraux H, Bertoncello N (2006) Subintimal angioplasty of tibial vessel occlusions in critical limb ischaemia: a good opportunity? Eur J Vasc Endovasc Surg 32(6):663-667

7. Met R, Van Lienden KP, Koelemay MJ et al (2008) Subintimal angioplasty for peripheral arterial occlusive disease: a systematic review. CardioVasc Interv Radiol 31(4):687-697
8. Reekers JA (2007) The feasibility of a percutaneous temporary pedal bypass. Eur J Vasc Endovasc Surg 34(1):50-52

9. Rutherford RB, Baker JD, Ernst C et al (1997) Recommend standards for reports dealing with lower extremity ischemia: revised version. J Vasc Surg 26(3):517-538

10. Reekers JA, Bolia AM (1998) Percutaneous intentional extraluminal (subintimal) recanalization: how to do it yourself. Eur $\mathbf{J}$ Radiol 28(3):192-198

11. Spaargaren GJ, Lee MJ, Reekers JA et al (2009) Evaluation of a new balloon catheter for difficult calcified lesions in infrainguinal arterial disease: outcome of a multicenter registry. CardioVasc Interv Radiol 32(1):132-135

12. Hirsch J, Warkentin TE, Raschke R et al (1998) Heparin and low molecular weight heparin. Chest 114(5):S489-S510

13. Sacks D, Marinelli DL, Martin LG et al (2003) Reporting standards for clinical evaluation of new peripheral arterial revascularization devices. J Vasc Interv Radiol 14(9; Pt 2):S395-S404

14. Paty PSK, Darling RCIII, Kreienberg PB et al (1998) Determinants of peroneal artery bypass failure. Vasc Endovasc Surg 32(6):603-608

15. Biancari F, Albäck A, Ihlberg L et al (1999) Angiographic runoff score as a predictor of outcome following femorocrural bypass surgery. Eur J Vasc Endovasc Surg 17(6):480-485

16. Desai TR, Meyerson SL, Skelly CL et al (2001) Patency and limb salvage after infrainguinal bypass with severely compromised ("blind") outflow. Arch Surg 136(6):635-642

17. Vraux H, Hammer F, Verhelst R et al (2000) Subintimal angioplasty of tibial vessel occlusions in the treatment of critical limb ischaemia: mid-term results. Eur $\mathrm{J}$ Vasc Endovasc Surg 20(5):441-446

18. Nydahl S, Hartshorne T, Bell PR et al (1997) Subintimal angioplasty of infrapopliteal occlusions in critically ischaemic limbs. Eur J Vasc Endovasc Surg 14(3):212-216

19. Giles KA, Pomposelli FB, Hamdan AD et al (2008) Infrapopliteal angioplasty for critical limb ischemia: relation of TransAtlantic InterSociety Consensus class to outcome in 176 limbs. J Vasc Surg 48(1):128-136

20. Ubbink DT, Vermeulen H, Spincemaille GH et al (2004) Systematic review and meta-analysis of controlled trials assessing spinal cord stimulation for inoperable critical leg ischaemia. Br J Surg 91(8):948-955

21. U.K. Severe Limb Ischaemia Study Group (1991) Treatment of limb threatening ischaemia with intravenous iloprost: a randomised double-blind placebo controlled study. Eur J Vasc Surg 5(5):511-516

22. Norgren L, Alwmark A, Angqvist KA et al (1990) A stable prostacyclin analogue (iloprost) in the treatment of ischaemic ulcers of the lower limb. A Scandinavian-Polish placebo controlled, randomised multicenter study. Eur J Vasc Surg 4(5):463467

23. The Oral Iloprost in Severe Leg Ischaemia Study Group (2000) Two randomised and placebo-controlled studies of an oral prostacyclin analogue (Iloprost) in severe leg ischaemia. Eur J Vasc Endovasc Surg 20(4):358-362

24. Sprengers RW, Lips DJ, Moll FL et al (2008) Progenitor cell therapy in patients with critical limb ischemia without surgical options. Ann Surg 247(3):411-420

25. Tateishi-Yuyama E, Matsubara H, Murohara T et al (2002) Therapeutic angiogenesis for patients with limb ischaemia by autologous transplantation of bone-marrow cells: a pilot study and a randomised controlled trial. Lancet 360(9331):427-435

26. Huang P, Li S, Han M et al (2005) Autologous transplantation of granulocyte colony-stimulating factor-mobilized peripheral blood mononuclear cells improves critical limb ischemia in diabetes. Diabetes Care 28(9):2155-2160 